\title{
Capacidade fotossintética de genótipos de amendoim em ambiente natural e controlado
}

\author{
Norma de Magalhães Erismann(1), Eduardo Caruso Machado(1) e Ignácio José de Godoy(1)
}

(1)Instituto Agronômico, Caixa Postal 28, CEP 13001-970 Campinas, SP. E-mail: normaeri@iac.sp.gov.br, caruso@iac.sp.gov.br, ijgodoy@iac.sp.gov.br

\begin{abstract}
Resumo - A capacidade fotossintética das cultivares de amendoim rasteiro (Arachis hypogaea L.) IAC-Caiapó e Runner IAC-886 foi avaliada sob condição controlada, em plantas cultivadas em vasos, mantidos em casa de vegetação, e sob condição natural, em plantas irrigadas, cultivadas em tanques de alvenaria. A resposta da taxa de assimilação líquida de $\mathrm{CO}_{2}(\mathrm{~A})$ em decorrência da densidade de fluxo de fótons fotossinteticamente ativos (DFFF) foi melhor em condição controlada, mas, nas duas condições, a mesma A máxima de ca. $28 \mu \mathrm{mol} \mathrm{m}^{-2} \mathrm{~s}^{-1}$ foi atingida. Em condição controlada, a saturação lumínica ocorreu próximo a $1.000 \mu \mathrm{mol} \mathrm{m}^{-2} \mathrm{~s}^{-1}$, ao passo que sob condição natural, ocorreu em DFFF maiores. A temperatura foliar entre 23 e $36^{\circ} \mathrm{C}$ não afetou A. A diferença de pressão de vapor entre a folha e o ar causou o fechamento parcial dos estômatos, diminuindo A, quando acima de $3,0 \mathrm{kPa}$. As capacidades fotossintéticas das duas cultivares de amendoim foram iguais. Ambas cultivares apresentaram boa adaptação às variações diárias do ambiente, ocorridas durante o verão, apresentando fotoinibição dinâmica da fotossíntese no início da tarde (13-14h), manifestada pela queda reversível da eficiência quântica máxima $\left(\mathrm{F}_{\mathrm{v}} / \mathrm{F}_{\mathrm{m}}\right)$ do fotossistema II.
\end{abstract}

Termos para indexação: Arachis hypogaea, fotossíntese, trocas gasosas, fluorescência da clorofila.

\section{Photosynthetic capacity of peanut genotypes under natural and controlled environment}

\begin{abstract}
Photosynthetic capacity of runner peanuts (Arachis hypogaea L.) cv. IAC-Caiapó and cv. Runner IAC-886 was evaluated under controlled condition, in plants grown on pots maintained in a greenhouse, and in irrigated plants grown on soil-filled tanks made of concrete, and exposed to natural ambient condition. $\mathrm{CO}_{2}$ net assimilation rate (A) response in relation to photosynthetic photon flux density (DFFF) was better in controlled condition, but in both conditions the same maximum A of ca. $28 \mu \mathrm{mol} \mathrm{m}^{-2} \mathrm{~s}^{-1}$ was reached. Under controlled condition, light saturation was about $1,000 \mu \mathrm{mol} \mathrm{m}^{-2} \mathrm{~s}^{-1}$, although under natural condition, saturation occurred at higher DFFF. Leaf temperature between 23 and $36^{\circ} \mathrm{C}$ did not affect $\mathrm{A}$, and leaf to air vapor pressure difference caused partial stomatal closing, decreasing A, when above $3.0 \mathrm{kPa}$. Photosynthetic capacities of the two cultivars were similar. Both cultivars showed good adaptation to daily changes of environmental conditions that occur during summer, showing dynamic photoinhibition of photosynthesis in the beginning of the afternoon (13-14h), as revealed by a reversible decrease in the maximum quantum efficiency $(\mathrm{Fv} / \mathrm{Fm})$ of photosystem II.
\end{abstract}

Index terms: Arachis hypogaea, photosynthesis, gas exchange, chlorophyll fluorescence.

\section{Introdução}

As cultivares de amendoim (Arachis hypogaea L.) do tipo Virginia (IAC-Caiapó e Runner IAC-886), padrão runner, além do alto potencial produtivo, possuem arquitetura de planta mais adequada para a colheita totalmente mecanizada. A cultivar Runner IAC-886 descende da cultivar multilinha Florunner, de origem americana, e é suscetível às doenças foliares, ao passo que a IAC-Caiapó apresenta resistência parcial e múltipla às mesmas doenças (Godoy et al., 1999).

Quando as doenças foliares são controladas, a produtividade da cultivar Runner IAC-886 supera à da IAC-Caiapó em 9 a 11\%. A duração do ciclo (130 dias) é geralmente menor e mais definida do que na cultivar IAC Caiapó (130-140 dias) (Godoy et al., 2003).

As condições ambientais para a produção de amendoim, em São Paulo, são tipicamente tropicais, 
recomendando-se seu cultivo na época de verão (Godoy et al., 2003). Nessa época, no meio do dia, a intensa radiação solar associada ou não à alta temperatura e ao deficit de pressão de vapor poderiam provocar fotoinibição dinâmica ou, eventualmente, crônica da fotossíntese, como descrito para outras espécies (Öquist et al., 1992; Long et al., 1994).

As mudanças na razão fonte-dreno também podem causar alterações de longo prazo nas taxas de fotossíntese das fontes. Em plantas de amendoim, Bagnall et al. (1988) sugeriram que em temperaturas subótimas $\left(15-20^{\circ} \mathrm{C}\right)$, o acúmulo de carboidratos nas folhas é o responsável pela diminuição da taxa de assimilação de $\mathrm{CO}_{2}(\mathrm{~A})$, podendo ser a causa principal da redução da taxa de crescimento das plantas.

No melhoramento de plantas, visando ao desenvolvimento de novas variedades de amendoim rasteiro, é interessante caracterizar e comparar as cultivares, atualmente disponíveis no mercado, quanto à capacidade fotossintética.

Este trabalho teve por objetivo avaliar as capacidades fotossintéticas das cultivares de amendoim rasteiro IAC-Caiapó e Runner IAC-886, em condições ambientais controladas e sob condições naturais de variações, típicas de dias claros de verão.

\section{Material e Métodos}

Ensaios experimentais com as cultivares de amendoim rasteiro IAC-Caiapó e Runner IAC-886 foram realizados no Centro Experimental de Campinas, do Instituto Agronômico (IAC), na estação quente e chuvosa, no ano agrícola de 2003/2004. As medidas, realizadas em condições naturais, foram feitas em plantas irrigadas, cultivadas em tanques de alvenaria, expostos às condições naturais. As sementes de cada cultivar foram plantadas em cinco tanques, medindo $2,0 \mathrm{~m}$ de comprimento por $0,5 \mathrm{~m}$ de largura e $0,6 \mathrm{~m}$ de profundidade, contendo terra e areia na proporção volumétrica de 2:1. Foi realizada a calagem com $180 \mathrm{~g} \mathrm{~m}^{-2}$ de calcário dolomítico e a adubação para reposição foi realizada por incorporação artificial $(10 \mathrm{~cm})$ na linha de plantio, com $15 \mathrm{~g} \mathrm{~m}^{-2}$ da fórmula NPK 8-28-16. As cultivares foram semeadas em 21/11/2003, numa linha central, em tanques alternados. Depois da emergência e do desbaste foram mantidas 20 plantas por tanque.

As determinações em condições ambientais controladas foram realizadas no laboratório em plantas cultivadas em vasos de $20 \mathrm{~L}$. As plantas foram irrigadas diariamente e mantidas em casa de vegetação até o dia de realização das medidas de trocas gasosas, quando foram transferidas para o laboratório. Ao substrato dos vasos, composto de partes iguais em volume de terra, areia e esterco, foram adicionados $30 \mathrm{~g}$ de calcário por vaso. Na semeadura em 17/12/2003, 5 g de superfosfato simples por vaso foram adicionados. Foram mantidos cinco vasos de cada cultivar, cada um com duas plantas.

Todas as variáveis de trocas gasosas, até mesmo a taxa de assimilação líquida de $\mathrm{CO}_{2}(\mathrm{~A})$ e de transpiração (E), a condutância estomática $\left(\mathrm{g}_{\mathrm{s}}\right)$, a concentração interna de $\mathrm{CO}_{2}(\mathrm{Ci})$, a diferença de pressão de vapor entre a folha e o ar ( $\left.\mathrm{DPV}_{\text {folha-ar }}\right)$, foram medidas com o equipamento portátil IRGA (Infra Red Gas Analyser), modelo LI-6400, que mede a concentração de $\mathrm{CO}_{2}$ e de vapor de água, por radiação infravermelha. O equipamento ainda mede, e permite controlar, o microclima e a densidade de fluxo de fótons fotossinteticamente ativos (DFFF) dentro da câmara de medida, que abrange uma área foliar de $6 \mathrm{~cm}^{2}(2 \times 3 \mathrm{~cm})$.

As medidas sob condições naturais foram efetuadas em folhas jovens, com idade entre 4 e 12 dias, totalmente expandidas, num folíolo da 2ª $3^{\text {a }}$ ou 4a folha, contadas a partir da extremidade do ramo. Uma determinação foi obtida mediante cinco repetições, em plantas diferentes do mesmo tanque, em folíolos diretamente expostos à radiação solar. A variação diária das trocas gasosas foi registrada em duas datas, 19/12/2003 e 14/1/2004, a primeira durante o período vegetativo, 28 dias após o plantio (dap), e a segunda, em plena floração (54 dap), em intervalos de aproximadamente uma hora, entre 5h45 e $17 \mathrm{~h}$.

A fim de homogeneizar as repetições, a DFFF foi gerada por um diodo emissor de luz acoplado à câmara de fotossíntese. No estádio vegetativo, as medidas foram feitas nas folhas totalmente expandidas da haste principal, que se desenvolve primeiro e é exclusivamente vegetativa. No estádio reprodutivo, foram selecionadas folhas dos ramos primários. A variação diária da fluorescência da clorofila a, do fotossistema II (FSII), foi medida em folíolos diretamente expostos à radiação solar, nas mesmas plantas (54 dap) e no mesmo dia $(14 / 1 / 2004)$ da segunda data de realização das médias de trocas gasosas. A fluorescência da clorofila a foi determinada, com um fluorômetro modulado (PAM-2000), de acordo com o método descrito por Maxwell \& Johnson (2000), utilizando a nomenclatura recomendada por Baker \& Rosenqvist (2004). A partir de medidas da fluorescência máxima $\left(F_{m}\right)$ e mínima $\left(F_{0}\right)$, 
em folíolos adaptados ao escuro, primeiro por uma noite e depois por 30 minutos, foi determinada a eficiência quântica máxima do FSII $\left(\mathrm{F}_{\mathrm{v}} / \mathrm{F}_{\mathrm{m}}\right.$, onde $\left.\mathrm{F}_{\mathrm{v}}=\mathrm{F}_{\mathrm{m}}-\mathrm{F}_{\mathrm{o}}\right)$. Na presença de radiação solar, foi novamente medida a fluorescência máxima $\left(\mathrm{F}_{\mathrm{m}}{ }^{\prime}\right)$, a mínima $\left(\mathrm{F}_{\mathrm{o}}{ }^{\prime}\right.$, após a excitação do FSI pela luz no vermelho distante) e a fluorescência no estado de equilíbrio dinâmico (F'), permitindo determinar a eficiência operacional $\left(\mathrm{F}_{\mathrm{q}}{ }^{\prime} / \mathrm{F}_{\mathrm{m}}{ }^{\prime}\right.$, onde $\mathrm{F}_{\mathrm{q}}{ }^{\prime}=\mathrm{F}_{\mathrm{m}}{ }^{\prime}-\mathrm{F}^{\prime}$ ) e a eficiência máxima do FSII $\left(F_{\mathrm{v}}{ }^{\prime} / \mathrm{F}_{\mathrm{m}}{ }^{\prime}\right.$, onde $\left.\mathrm{F}_{\mathrm{v}}{ }^{\prime}=\mathrm{F}_{\mathrm{m}}{ }^{\prime}-\mathrm{F}_{\mathrm{o}}{ }^{\prime}\right)$. $\mathrm{O}$ fluxo alternativo de elétrons (FAE) foi estimado, sem considerar a respiração mitocondrial, pela razão: $\mathrm{FAE}=\left(\mathrm{F}_{\mathrm{q}}{ }^{\prime} / \mathrm{F}_{\mathrm{m}}{ }^{\prime}\right) /(\mathrm{A} / 0,84 \mathrm{DFFF})$, em que 0,84 é o coeficiente de absorção da luz pela folha (Edwards \& Baker, 1993).

Os estudos do efeito da luz, de concentração interna de $\mathrm{CO}_{2}$ (Ci) e da temperatura, nas trocas gasosas, foram realizados em folhas jovens de plantas cultivadas em vasos, no estádio reprodutivo (71 a 82 dap), correspondente ao final da floração e plena formação de vagens. As curvas de resposta de $\mathrm{A}$ à luz $\left(\mathrm{CO}_{2}\right.$ controlado em $38 \mathrm{~Pa}$ ) e de A em função de Ci (DFFF de $1.250 \mu \mathrm{mol} \mathrm{m}^{-2} \mathrm{~s}^{-1}$ ) foram realizadas em câmara de crescimento (KTLK 1600), depois da aclimatização, pelo período mínimo de uma hora, em condições controladas de temperatura, DFFF e DPV $\left(30^{\circ} \mathrm{C}, 300 \mu \mathrm{mol} \mathrm{m}^{-2} \mathrm{~s}^{-1} \mathrm{e}\right.$ $1,0 \mathrm{kPa}$, respectivamente), mediante quatro repetições, em plantas diferentes. A DPV $V_{\text {folha-ar }}$ permaneceu próxima a $1,0 \mathrm{kPa}$, controlando-se a pressão de vapor da água no ar de entrada, com o auxílio de um medidor de ponto de orvalho (LI-610), acoplado ao IRGA LI-6400. A variação de Ci foi obtida pelo controle do $\mathrm{CO}_{2}$, entre 5 e $140 \mathrm{~Pa}$, do ar de entrada (referência) da câmara de medida, do IRGA LI-6400. A partir dos dados de A, foi estimada uma medida relativa da limitação estomática da fotossíntese $(l, \%)$, pela relação: $l=100(\mathrm{ACi}-\mathrm{ACe}) / \mathrm{ACi}$, em que: ACe é a assimilação líquida real, quando a concentração do $\mathrm{CO}_{2}$ no ambiente externo (referência) é 40 Pa, e Aci é a assimilação líquida ideal (resistência à difusão do $\mathrm{CO}_{2}=0$ ), assumindo que a concentração interna do $\mathrm{CO}_{2}(\mathrm{Ci})$ seja de $40 \mathrm{~Pa}$ (Long \& Bernacchi, 2003).

Os dados da curva de resposta de A em função da DFFF, de 2.000 até $0 \mu \mathrm{mol} \mathrm{m}{ }^{-2} \mathrm{~s}^{-1}$, foram ajustados pela equação $A=A_{\text {máx }}\left[1-e^{-k(D F F F-I c)}\right]$, que permite estimar a assimilação líquida máxima $\left(\mathrm{A}_{\text {máx }}\right)$, em que: $\mathrm{k}$ é uma constante e $\mathrm{I}_{\mathrm{C}}$ é o ponto de compensação lumínica (Prado \& Moraes, 1997).

No ensaio da resposta de A em função da temperatura foliar foi realizada uma série de medidas num mesmo folíolo, sob DFFF saturante de $1.250 \mu \mathrm{mol} \mathrm{m}^{-2} \mathrm{~s}^{-1} \mathrm{e}$ $\mathrm{DPV}_{\text {folha-ar }}$ de ca. $1,5 \mathrm{kPa}$, submetido a temperaturas crescentes, controladas pela temperatura do bloco metálico, de 20 a $40^{\circ} \mathrm{C}$, da câmara de medida, do IRGA LI-6400.

\section{Resultados e Discussão}

A Figura 1 mostra a variação diária da temperatura foliar, da $\mathrm{DPV}_{\text {folha-ar }}$ e da DFFF, registrada durante a execução das medidas de trocas gasosas (Figura 2) em condições naturais, nas cultivares de amendoim, no estádio vegetativo e em plena floração. Os valores de $\mathrm{DPV}_{\text {folha-ar }}$ permaneceram baixos (0,8 a 2,2 kPa) durante todo o segundo dia de medidas em 14/1/2004, mas na primeira data, 19/12/2003, os valores de $\mathrm{DPV}_{\text {folha-ar }}$ aumentaram a partir das $10 \mathrm{~h}$, acompanhando o aumento da temperatura foliar, atingindo picos de $\mathrm{DPV}_{\text {folha-ar }}$ acima de 3,5 kPa, no início da tarde, para a cultivar de amendoim Runner IAC-886 e, no final da tarde, para IAC-Caiapó. Nas duas datas de realização das medidas, a menor temperatura foliar $\left(21\right.$ e $23^{\circ} \mathrm{C}$, respectivamente) foi registrada no início do dia. No meio do dia e durante toda a tarde, as temperaturas foliares foram superiores a $30^{\circ} \mathrm{C}$, atingindo valores máximos de $36-37^{\circ} \mathrm{C}$, às $14 \mathrm{~h}$ da primeira data, e de $35^{\circ} \mathrm{C}$, às 12h30 da segunda data.

Os cursos diários de $\mathrm{A}, \mathrm{g}_{\mathrm{s}}, \mathrm{E}$ (Figura 2), Ci e da eficiência do uso da água (A/E) (Figura 3), das duas cultivares de amendoim, foram muito semelhantes entre si, sobretudo na segunda data. Nos dois dias de medidas, A aumentou rapidamente, nas primeiras horas da manhã (até 8h), acompanhando o aumento da DFFF até $1.000 \mu \mathrm{mol} \mathrm{m}{ }^{-2} \mathrm{~s}^{-1}$ (Figura 1), permanecendo estável ou subindo um pouco mais até $12 \mathrm{~h} 30$ (DFFF máxima de $2.000 \mu \mathrm{mol} \mathrm{m}^{-2} \mathrm{~s}^{-1}$ ). No início da tarde, ao redor das $14 \mathrm{~h}$, foi notada uma baixa na curva de A, apesar dos valores saturantes de DFFF deste horário, com tendência à recuperação às 15h. A diminuição de A depois das $16 \mathrm{~h}$ acompanha a baixa luminosidade da tarde. $\mathrm{O}$ valor máximo de $\mathrm{A}$, medido no estádio vegetativo, foi de $21 \mu \mathrm{mol} \mathrm{m}{ }^{-2} \mathrm{~s}^{-1}$, às $12 \mathrm{~h} 45$, ao passo que, em plena floração, foi de 28 a $29 \mu \mathrm{mol} \mathrm{m}^{-2} \mathrm{~s}^{-1}$, às $11 \mathrm{~h} 20$. Estes valores de A máxima estão dentro do intervalo de 13 a $41 \mu \mathrm{mol} \mathrm{m}{ }^{-2} \mathrm{~s}^{-1}$, relatado por Ketring et al. (1982), que reuniu dados de vários trabalhos de $\mathrm{A}$ em folhas de amendoim.

As respostas diárias de $\mathrm{g}_{\mathrm{s}}$ e $\mathrm{E}$ acompanharam as variações de A. Os valores de E aumentaram com o 
aumento da temperatura foliar até o meio do dia; os valores máximos de A/E (Figura 3 C e D) foram observados no início da manhã. No período das $9 \mathrm{~h}$ às $12 \mathrm{~h} 30$, as plantas em plena floração apresentaram valores de $\mathrm{A}, \mathrm{g}_{\mathrm{s}}$ e $\mathrm{E}$ maiores que as plantas no estádio vegetativo. É provável que esta diferença de resposta, observada em estádios fisiológicos distintos, se deva à maior exigência por fotoassimilados, que ocorre durante o florescimento. Existem evidências de que os valores de A de folhas individuais são controlados pela necessidade do dreno em cultivares comerciais de amendoim, indicando que o acúmulo de carboidratos na folha inibe a fotossíntese (Bagnall et al., 1988).

Uma diferença de resposta, entre as duas cultivares, foi notada na primeira data de medidas, em horários da tarde. Entre 13 e 14h foram obtidos valores de A, gs e E maiores para a cultivar IAC-Caiapó e, no final da tarde, esta tendência se inverteu, com a cultivar Runner IAC-886 apresentando uma resposta melhor. Uma das causas dessa diferença parece estar associada a variações de

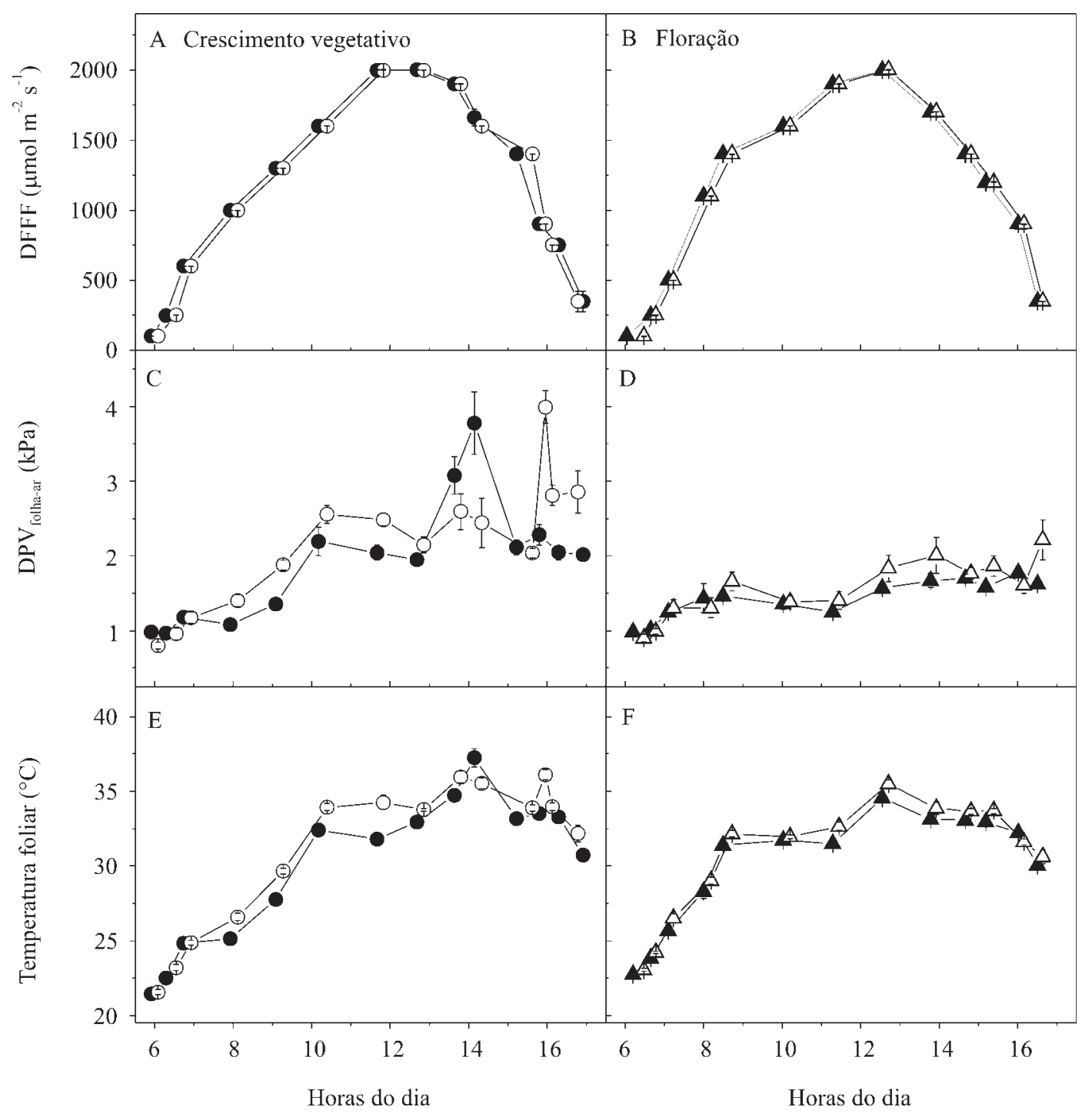

Figura 1. Curso diário da densidade de fluxo de fótons fotossinteticamente ativos (DFFF), da diferença de pressão de vapor entre a folha e o ar $\left(\mathrm{DPV}_{\text {folha-ar }}\right)$ e da temperatura foliar, nas cultivares de amendoim Runner IAC-886 (símbolos cheios) e IAC-Caiapó (símbolos vazios), no estádio vegetativo(•, ○) e em plena floração $(\boldsymbol{\Lambda}, \triangle)$, sob condições naturais. Cada ponto representa o valor médio ( \pm erro-padrão) de cinco repetições, em plantas diferentes. 
$\mathrm{DPV}_{\text {folha-ar, }}$ pois o fechamento estomático ( $\mathrm{g}_{\mathrm{s}}$ de $0,5-0,4$ para $0,2-0,1 \mathrm{~mol} \mathrm{~m}^{-2} \mathrm{~s}^{-1}$ ) ocorreu, em cada cultivar, nos horários correspondentes aos valores de $\mathrm{DPV}_{\text {folha-ar }}$ acima de 3,0 kPa (Figura 1).

Nas plantas em plena floração, a baixa observada nas curvas de trocas gasosas, depois do meio-dia, parece não estar relacionada com a $\mathrm{DPV}_{\text {folha-ar, }}$ que permaneceu baixa $(0,8$ a 2,2 $\mathrm{kPa})$ durante todo o dia. O fechamento estomático ( $g_{\mathrm{s}}$ de $0,9-0,8$ para $0,6-05 \mathrm{~mol} \mathrm{~m}^{-2} \mathrm{~s}^{-1}$ ) nesse horário, provavelmente, foi suficiente para limitar
E, mas não A. Em ensaios realizados em condições controladas, os valores de $g_{s}$ não passaram de $0,6 \mathrm{~mol} \mathrm{~m}^{-2} \mathrm{~s}^{-1}$. Além disso, como conseqüência da limitação estomática de A, Ci deve diminuir (Long \& Bernacchi, 2003). Esse fato foi observado no primeiro dia de medidas (Figura $3 \mathrm{~A}$ ), quando $\mathrm{DPV}_{\text {folha-ar }}$ foi acima de $3,0 \mathrm{kPa}$, mas não na outra data (Figura $3 \mathrm{~B}$ ), que mostra que $\mathrm{Ci}$ permaneceu estável das 7 às $16 \mathrm{~h}$.

Os dados de A dos dois dias de medidas, obtidos em condições próximas à saturação lumínica

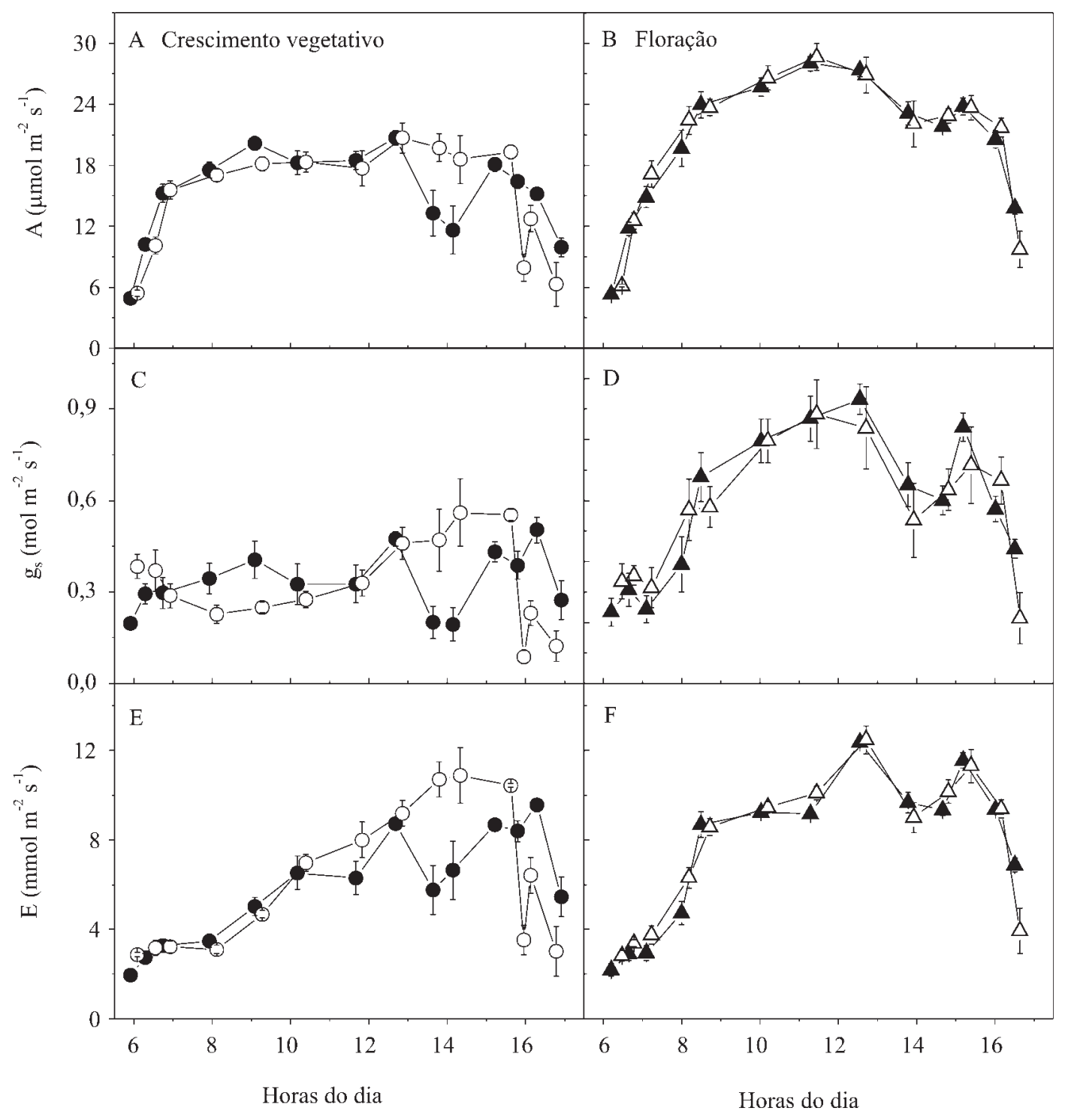

Figura 2. Curso diário da taxa de assimilação líquida de $\mathrm{CO}_{2}(\mathrm{~A})$, condutância estomática (gs) e da taxa de transpiração (E) nas cultivares de amendoim Runner IAC-886 (símbolos cheios) e IAC-Caiapó (símbolos vazios), no estádio vegetativo $(\bullet, \bigcirc)$ e em plena floração $(\boldsymbol{\Lambda}, \Delta)$. Cada ponto representa o valor médio ( \pm erro-padrão) de cinco repetições, em plantas diferentes. 
(DFFF $\geq 900 \mu \mathrm{mol} \mathrm{m} \mathrm{m}^{-2} \mathrm{~s}^{-1}$ ), foram reunidos e apresentados em decorrência da $D P V_{\text {folha-ar }}$ e da temperatura foliar (Figura 4). Os dados apresentaram um agrupamento de acordo com o dia de realização das medidas, e os pontos referentes aos menores valores correspondem às medidas obtidas durante o estádio vegetativo. Observou-se diminuição de $A$ apenas quando a $\mathrm{DPV}_{\text {folha-ar }}$ foi superior a 3,0 (Figura $4 \mathrm{~A}$ ).

Apesar da dispersão dos dados obtidos em condições naturais, nenhuma tendência de aumento ou diminuição de A foi verificada com a variação da temperatura foliar, de 23 a $36^{\circ} \mathrm{C}$ (Figura 4 B). Além disso, os valores de A, de 24-25 $\mu \mathrm{mol} \mathrm{m}{ }^{-2} \mathrm{~s}^{-1}$, obtidos em condições controladas de DFFF e DPV folha-ar (Figura 4 B), permaneceram estáveis com o aumento da temperatura foliar. Pontos referentes à $D P V_{\text {folha-ar }}$ maior que $3,0 \mathrm{kPa}$ foram discriminados, pois, a diminuição de $\mathrm{A}$, neste caso, se deve provavelmente ao efeito da $D P V_{\text {folha-ar }}$ e não à elevação da temperatura foliar.
Vu (2005) também não observou diferenças entre os valores de A de plantas de amendoim aclimatizadas a temperaturas de 1,5 e $6,0^{\circ} \mathrm{C}$ acima da temperatura ambiente (ca. $35^{\circ} \mathrm{C}$ ), relatando valores de A de 32,4 e $34,2 \mu \mathrm{mol} \mathrm{m}{ }^{-2} \mathrm{~s}^{-1}$, respectivamente, sob condições controladas de $\mathrm{CO}_{2}$ em $360 \mu \mathrm{mol} \mathrm{mol}^{-1}$. Bagnall et al. (1988) mostraram que as respostas de A de folhas individuais de amendoim são controladas pelo dreno; observaram pouca diferença na resposta imediata de A, quando a maior parte da planta (dreno) foi mantida a $30^{\circ} \mathrm{C}$ e a temperatura da folha (fonte) variou entre $15 \mathrm{e}$ $30^{\circ} \mathrm{C}$. Nessas condições, A diminuiu pouco (17\%), quando a temperatura foliar foi de $19^{\circ} \mathrm{C}$ ou inferior, ao passo que o resfriamento $\left(19^{\circ} \mathrm{C}\right)$ da maior parte da planta (dreno), por três dias, causou declínio de 70\% em A das folhas (fonte) mantidas a $30^{\circ} \mathrm{C}$.

Ensaios de trocas gasosas, realizados em condições controladas (Figura 5), também não mostraram nenhuma diferença entre as capacidades fotossintéticas das duas

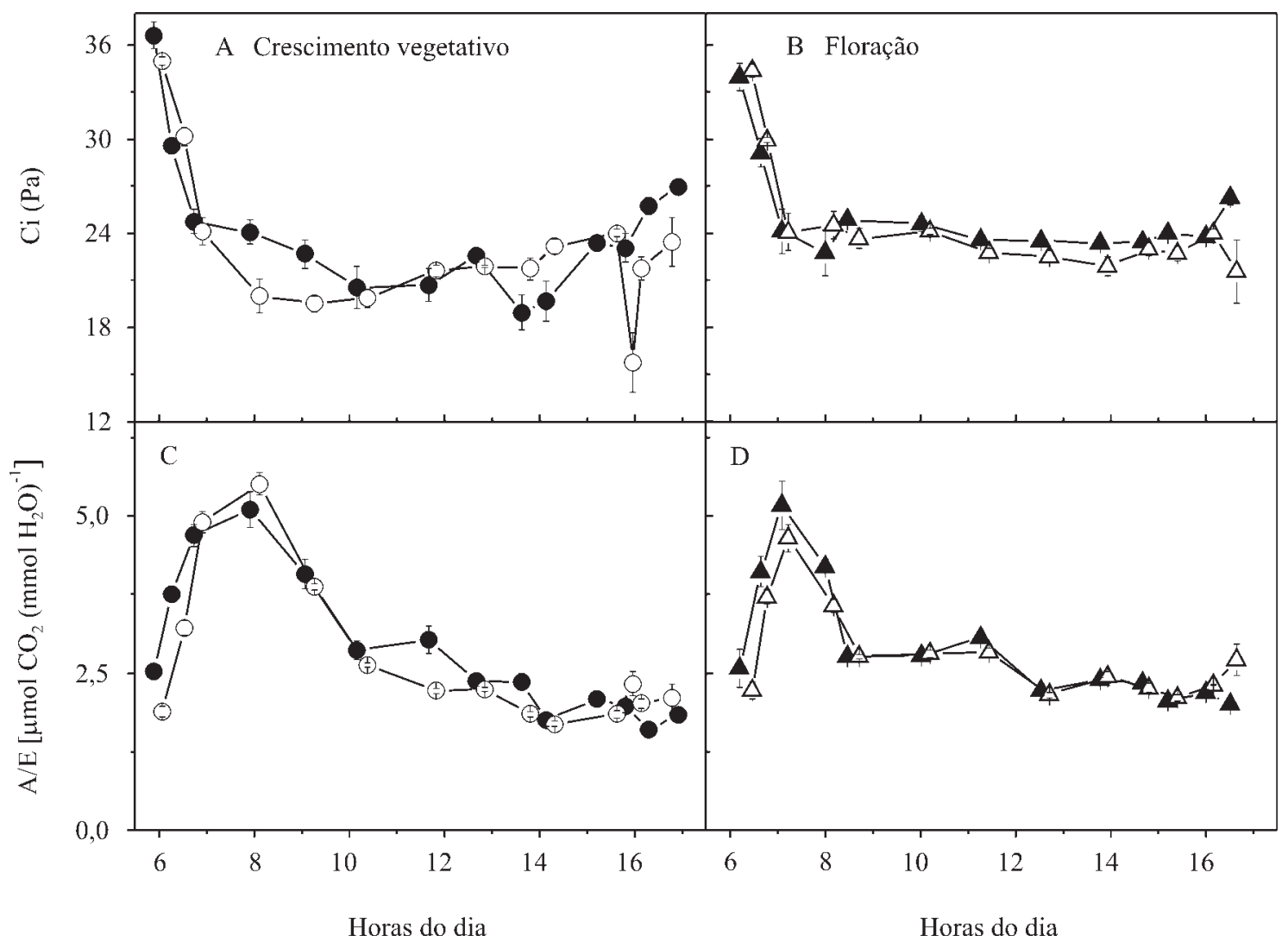

Figura 3. Curso diário da concentração interna de $\mathrm{CO}_{2}(\mathrm{Ci})$ e da eficiência do uso da água (A/E), nas cultivares de amendoim Runner IAC-886 (símbolos cheios) e IAC-Caiapó (símbolos vazios), no estádio vegetativo $(\bullet, \bigcirc)$ e em plena floração $(\boldsymbol{\Delta}, \Delta)$. Cada ponto representa o valor médio ( \pm erro-padrão) de cinco repetições, em plantas diferentes. 
cultivares. Assim, dados referentes às duas cultivares foram reunidos e analisados juntos.

A resposta de A em função de Ci (Figura 5 B) mostra que A aumentou com o crescimento dos níveis de $\mathrm{Ci}$, como esperado para uma planta $\mathrm{C}_{3}$ típica (Long \& Bernacchi, 2003). O valor máximo de Ade $26 \mu \mathrm{mol} \mathrm{m}^{-2} \mathrm{~s}^{-1}$, observado em condições ambientais normais, foi para $39 \mu \mathrm{mol} \mathrm{m}^{-2} \mathrm{~s}^{-1}$, em Ci de $58 \mathrm{~Pa}$, aumentando pouco em níveis ainda mais altos de $\mathrm{Ci}$. O ponto de compensação de $\mathrm{CO}_{2}$, calculado a partir da porção linear do início da curva, foi de 6,4 Pa, próximo aos valores referentes a cultivares comercias de amendoim (Ketring et al., 1982). A eficiência de carboxilação, alcançada pela inclinação da reta inicial (Long \& Bernacchi, 2003), foi de 1,85 $\mu \mathrm{mol} \mathrm{m}^{-2} \mathrm{~s}^{-1} \mathrm{~Pa}^{-1}$. Ainda a partir desta curva, a limitação imposta pelo estômato na fotossíntese (l) foi estimada em 22,9\%, determinada sob condições ótimas de temperatura e DFFF, quando $g_{s}$ permaneceu entre 0,35 e 0,53.

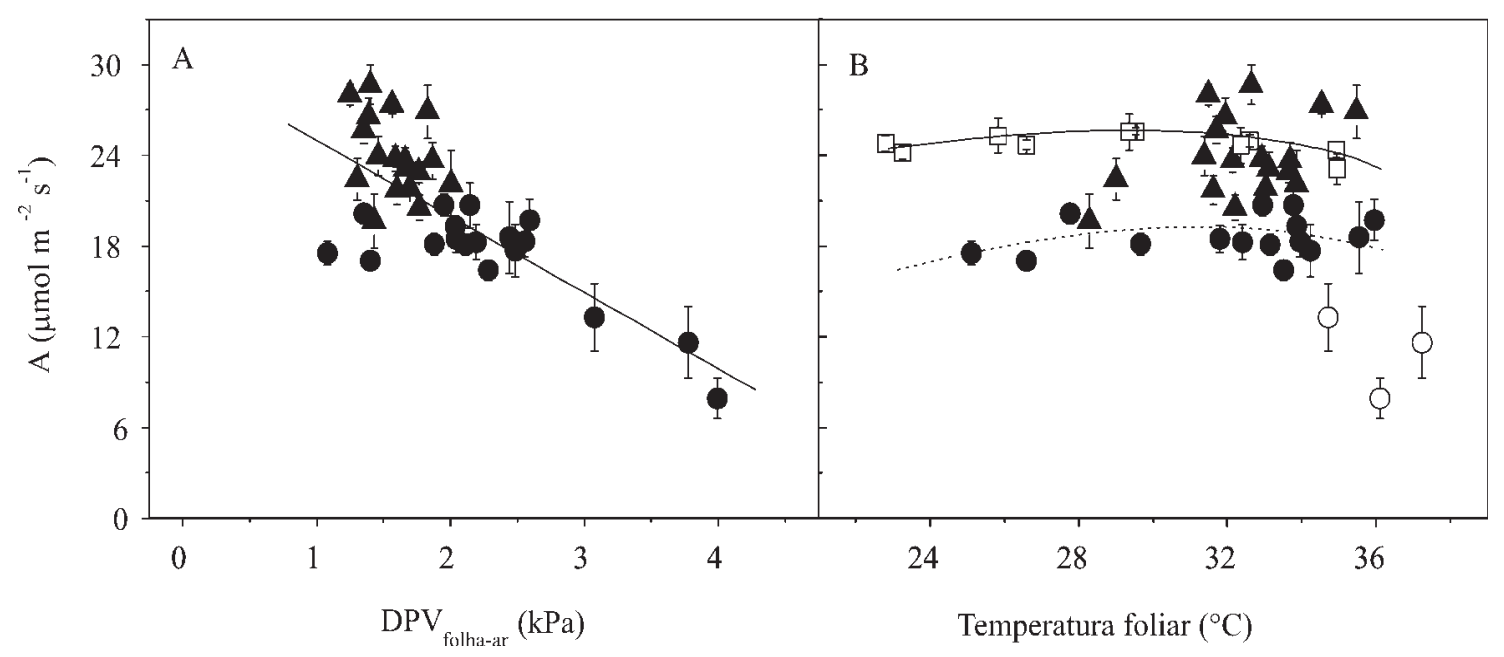

Figura 4. Taxa de assimilação líquida de $\mathrm{CO}_{2}(\mathrm{~A})$ em função da diferença de pressão de vapor entre a folha e o ar (DPV $\left.V_{\text {folha-ar }}\right)$ e da temperatura da folha, nas cultivares de amendoim, no estádio vegetativo (๑) e de florescimento ( $\mathbf{\Delta})$. Os pontos obtidos em $\mathrm{DPV}_{\text {folha-ar }}$ maior que 3,0 (O) e as medidas realizadas em condições controladas $(\square)$ estão indicados. Cada ponto representa o valor médio de cinco $(\bullet, \bigcirc, \boldsymbol{\Delta})$ ou de três $(\square)$ repetições ( \pm erro-padrão), em plantas diferentes.
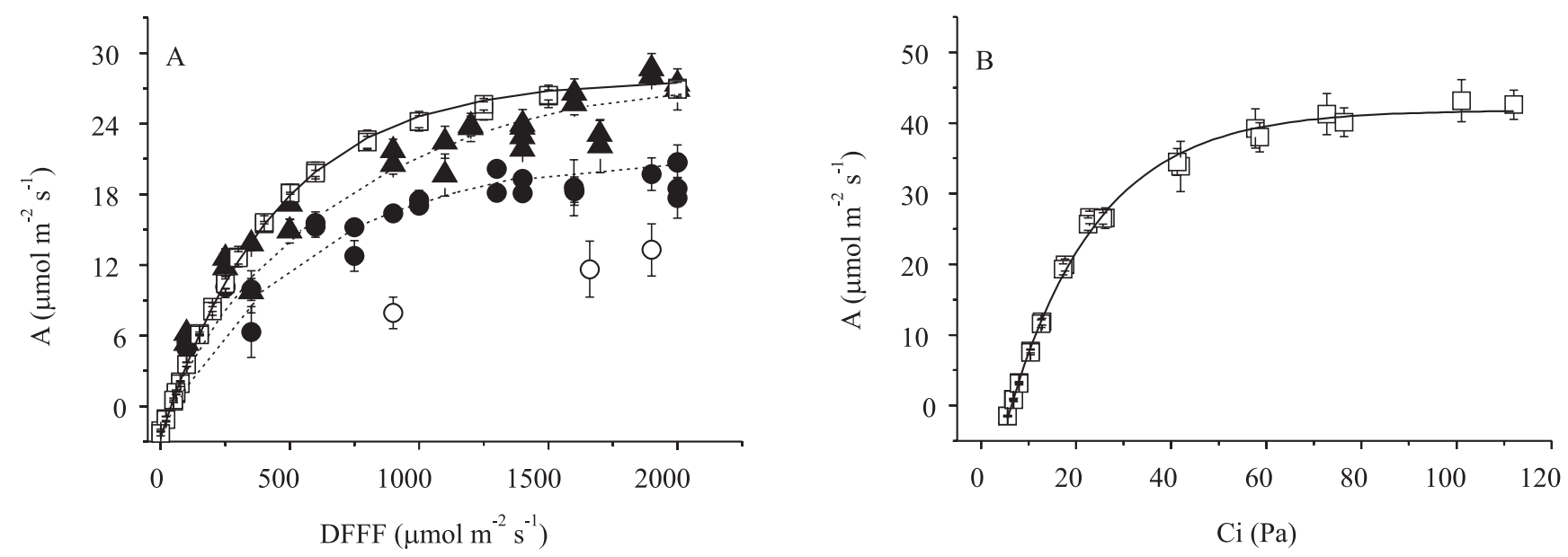

Figura 5. Taxa de assimilação líquida de $\mathrm{CO}_{2}$ (A) em função da densidade de fluxo de fótons fotossinteticamente ativos (DFFF) e da concentração interna de $\mathrm{CO}_{2}(\mathrm{Ci})$, nas cultivares de amendoim, sob condições controladas $(\square)$ e sob condições naturais, no estádio vegetativo (๑) e de florescimento $(\mathbf{\Delta})$. Os pontos obtidos com DPV folha-ar maior que 3,0 estão indicados (O). Cada ponto representa o valor médio de cinco $(\mathbf{\bullet}, \bigcirc, \mathbf{\Lambda})$ ou de quatro $(\square)$ repetições ( \pm erro-padrão), em plantas diferentes. 
A resposta de A em função de DFFF, obtida em condições ambientais ótimas $\left(30^{\circ} \mathrm{C}, \mathrm{DPV}_{\text {follha-ar }}=1,0 \mathrm{kPa}\right)$, parece ser ligeiramente melhor do que a observada em condições naturais, com as plantas em floração (Figura 5 A). Em condições controladas, foi calculado

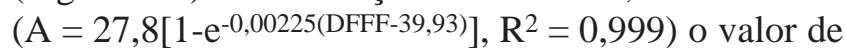
$27,8 \mu \mathrm{mol} \mathrm{m}^{-2} \mathrm{~s}^{-1}$ para A máxima, e 39,9 $\mu \mathrm{mol} \mathrm{m}^{-2} \mathrm{~s}^{-1}$ para o ponto de compensação lumínica. A saturação lumínica ocorreu ao redor de $1.000 \mu \mathrm{mol} \mathrm{m}^{-2} \mathrm{~s}^{-1}$. Estes valores estão de acordo com o descrito anteriormente (Ketring et al., 1982) para diferentes genótipos de amendoim. O rendimento quântico máximo aparente, obtido pela inclinação da reta da porção inicial da curva, foi de $0,055 \mu \mathrm{mol} \mathrm{CO}{ }_{2} \mathrm{mmol}^{-1}$.
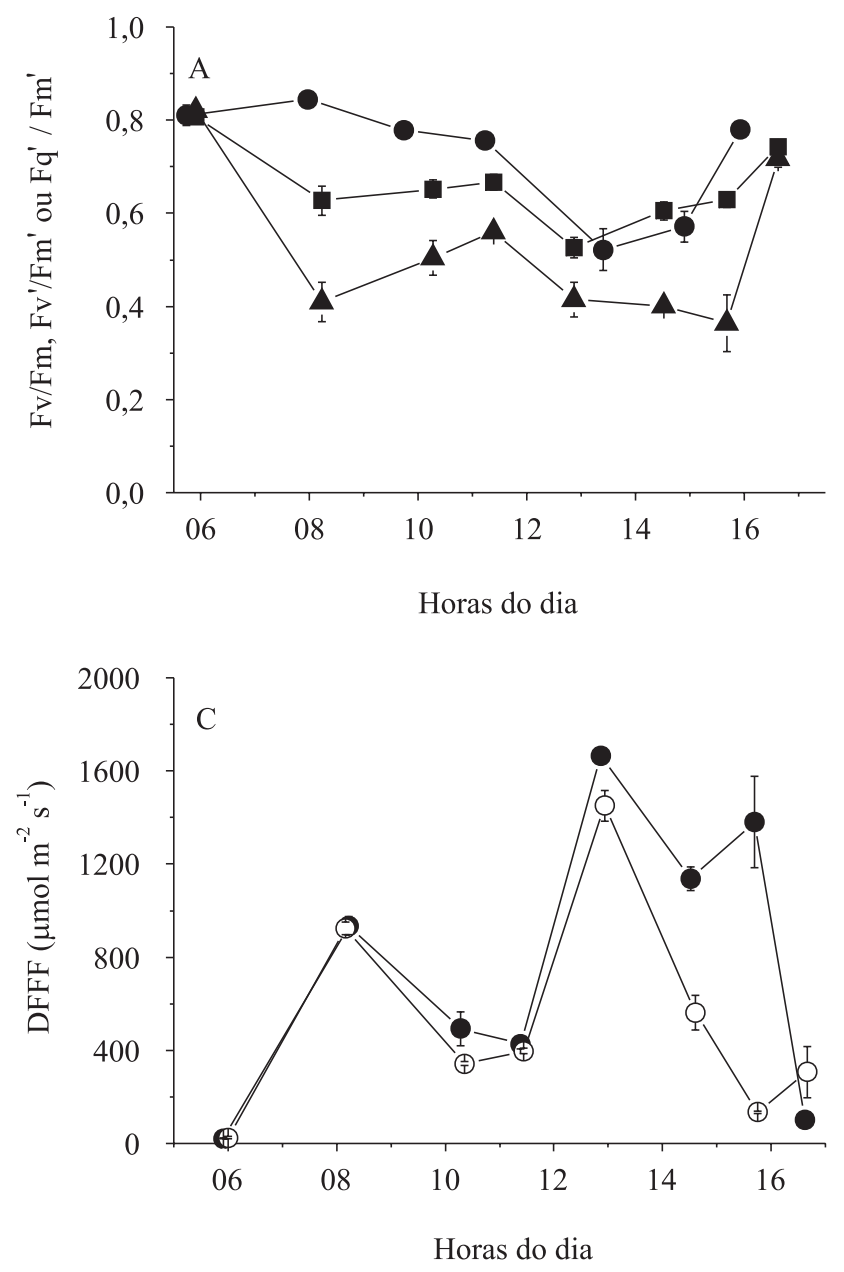

A curva de luz obtida em condições naturais (com as plantas em floração), quando comparada com a curva obtida em condições controladas, sugere a ocorrência de fotoinibição dinâmica ou fotoprotetora da fotossíntese (Long et al., 1994). A máxima tende ao mesmo valor, nas duas condições, ao passo que a eficiência quântica aparente de assimilação de $\mathrm{CO}_{2}$, avaliada pela inclinação da reta inicial das duas curvas, parece diminuir (Figura 5 A), e a saturação lumínica tende a ocorrer numa DFFF maior, próxima à radiação solar máxima, em condições naturais.

As medidas de fluorescência da clorofila a, realizadas nas cultivares de amendoim Runner IAC-886 (Figura 6 A) e IAC-Caiapó (Figura 6 B), permitiram
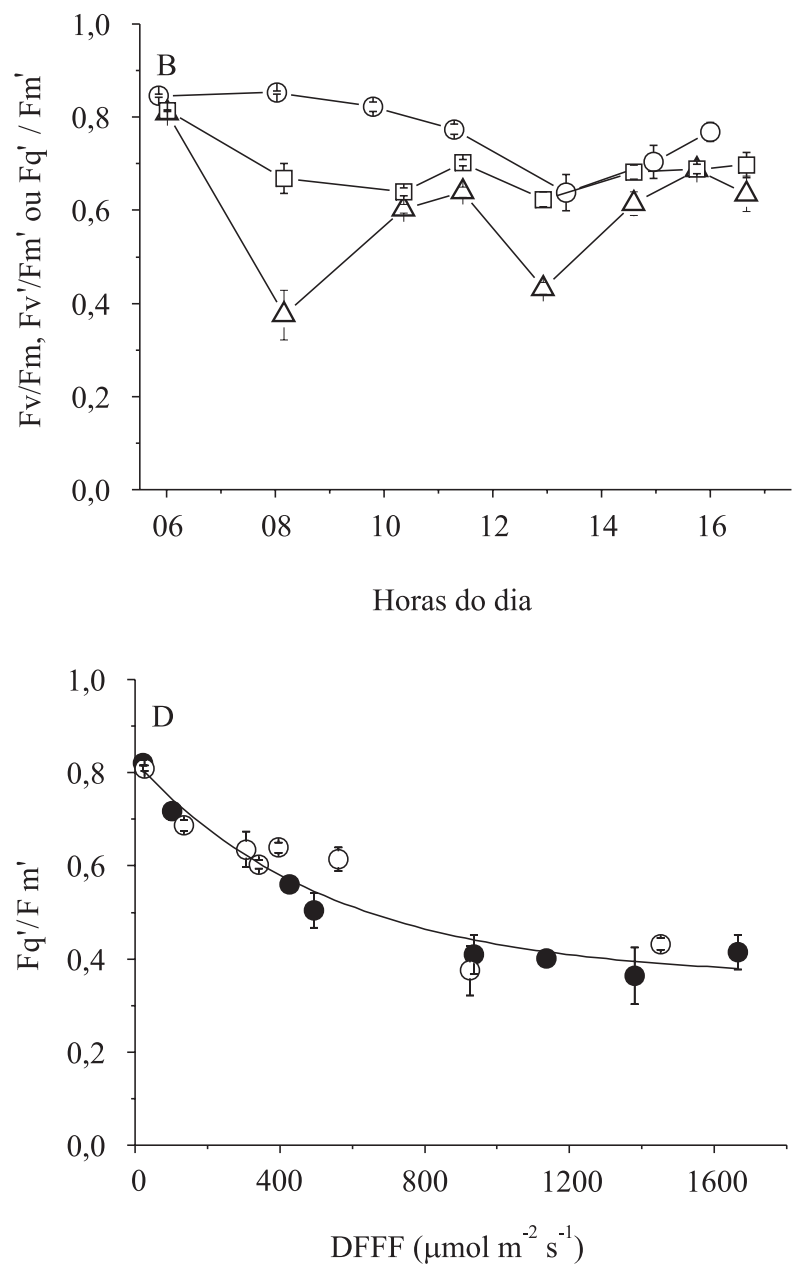

Figura 6. Curso diário da eficiência quântica máxima $\left(F_{v} / F_{m}: \bullet\right.$ em $A, \bigcirc$ em B), da eficiência máxima $\left(F_{v}{ }^{\prime} / F_{m}{ }^{\prime}: \square, \square\right)$ e da eficiência operacional $\left(\mathrm{F}_{\mathrm{q}}{ }^{\prime} / \mathrm{F}_{\mathrm{m}}{ }^{\prime}: \boldsymbol{\Delta}, \Delta\right)$ do FSII, e densidade de fluxo de fótons fotossinteticamente ativos (DFFF) da radiação solar (C) registrada durante as medidas, nas cultivares de amendoim Runner IAC-886 (A e símbolos cheios) e IAC-Caiapó (B e símbolos vazios), sob condições naturais. Em D, eficiência operacional $\left(\mathrm{F}_{\mathrm{q}}{ }^{\prime} / \mathrm{F}_{\mathrm{m}}{ }^{\prime}\right)$ do FSII em função da DFFF. Cada ponto representa 0 valor médio ( \pm erro-padrão) de seis repetições, em plantas diferentes. 
acompanhar a eficiência do transporte de elétrons do FSII, durante o mesmo dia das medidas das trocas gasosas, com as plantas em plena floração (54 dap). A ocorrência de fotoinibição dinâmica, manifestada pela queda diária reversível da eficiência quântica máxima do FSII $\left(F_{v} / F_{m}\right)$ (Öquist et al., 1992; Long et al., 1994), foi verificada durante o início da tarde (13-15h), logo após o horário (12h30) de maior calor e DFFF, que também coincide com a baixa observada nas curvas de trocas gasosas (Figura $2 \mathrm{~B}, \mathrm{D}$ e F).

$O$ valor de $F_{v} / F_{m}$ permaneceu em cerca de 0,8 até as $10 \mathrm{~h}$, diminuiu para $0,5-0,6$ nas horas de maior excesso de energia luminosa (13-14h) e, no final da tarde, retornou a valores próximos ao do começo do dia. Essa fotoinibição dinâmica ou protetora do FSII tem sido freqüentemente correlacionada com o aumento da formação de zeaxantina e ao aumento da dissipação de energia térmica do FSII, promovidos pelo gradiente de $\mathrm{pH}$ transtilacóide gerado pelo transporte de elétrons (Long et al., 1994; Maxwell \& Johnson, 2000).

A fotoinibição parece ser conseqüência inevitável do funcionamento do FSII, quando a luz causa o fechamento prolongado de mais de $40 \%$ dos centros de reação do FSII (Öquist et al., 1992). A queda diária da eficiência operacional $\left(\mathrm{F}_{\mathrm{q}}{ }^{\prime} / \mathrm{F}_{\mathrm{m}}{ }^{\prime}\right)$, ao longo do dia, foi maior do que da eficiência máxima $\left(\mathrm{F}_{\mathrm{v}}{ }^{\prime} / \mathrm{F}_{\mathrm{m}}{ }^{\prime}\right)$, com todos os centros do FSII abertos (Figura 6 A e B), mostrando um fechamento parcial ( $\leq 44 \%$ ) dos centros do FSII, ao longo do dia. Processos rápidos, não fotoquímicos, de dissipação da energia de excitação, induzidos por alto gradiente de $\mathrm{pH}$, também contribuíram para diminuir $\mathrm{F}_{\mathrm{q}}{ }^{\prime} / \mathrm{F}_{\mathrm{m}}$ ' (Maxwell \& Johnson, 2000). Entretanto, tais mecanismos de regulação da fotossíntese devem ter conferido proteção aos centros de reação do FSII (Öquist et al., 1992), evitando a inativação irreversível, nas cultivares de amendoim expostas às condições ambientais naturais de dias claros de verão.

$\mathrm{O}$ curso diário de $\mathrm{F}_{\mathrm{q}}{ }^{\prime} / \mathrm{F}_{\mathrm{m}}$ ' do FSII (Figura $6 \mathrm{~A}$ e B) também mostra que, em determinados horários (15-16h), ocorreram diferenças significativas entre as duas cultivares. Mas as variações, observadas nos valores de $\mathrm{F}_{\mathrm{q}}{ }^{\prime} / \mathrm{F}_{\mathrm{m}}$ ' ao longo do dia, parecem estar relacionadas às variações da DFFF incidente, no instante de realização das medidas, pois, tais medidas foram realizadas em condições naturais de DFFF (Figura 6 C), em que a queda de DFFF nos horários das 10-11h e das 15-16h (para IAC-Caiapó) correspondeu à passagem de nuvens. A queda de Fq'/Fm' acompanhou aumento da
DFFF, atingindo valores mínimos (ca. 0,4) e estáveis em DFFF acima de $800 \mu \mathrm{mol} \mathrm{m}^{-2} \mathrm{~s}^{-1}$ (Figura $6 \mathrm{D}$ ). Nesse caso, a reposta de $\mathrm{F}_{\mathrm{q}}$ '/ $\mathrm{F}_{\mathrm{m}}$ ' das duas cultivares de amendoim, quando submetidas às mesmas condições de DFFF, foram semelhantes.

Em ambas cultivares, A apresentou pequenas variações entre $8 \mathrm{~h} 30$ e $16 \mathrm{~h}$, ainda que a DFFF e a temperatura nesse período tenham aumentado (Figuras 1 e 2). No mesmo período, Fq'/Fm' permaneceu estável a partir de $800 \mu \mathrm{mol} \mathrm{m}^{-2} \mathrm{~s}^{-1}$ (Figura 6 D), indicando que, acima desta DFFF, ocorreu aumento no fluxo de elétrons através do FSII. Uma vez que o correspondente acréscimo em A foi pequeno, os elétrons em excesso, provavelmente, foram utilizados em rotas metabólicas alternativas, onde a fotorrespiração parece ser a principal (Cornic \& Fresneau, 2002), como também foi observado por Ribeiro et al. (2004) em feijoeiro. De fato, o FAE às 8h foi, respectivamente, 19,5 $\pm 1,9$ e 16,8 $\pm 1,1$ em Runner IAC-886 e IAC-Caiapó,

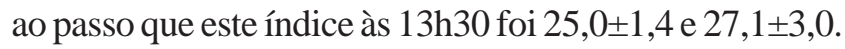

O aumento da relação entre a eficiência quântica operacional e eficiência quântica de redução do $\mathrm{CO}_{2}$ indica incremento no dreno de elétrons para fotorrespiração (Edwards \& Baker, 1993; Cornic \& Fresneau, 2002). Em conseqüência da fotorrespiração, mais $\mathrm{CO}_{2}$ é liberado, podendo causar diminuição na taxa de assimilação líquida de $\mathrm{CO}_{2}$. Entretanto, como houve pequena variação em $\mathrm{A}$, no período considerado, parte do excesso de elétrons também deve ter sido utilizada na própria assimilação de $\mathrm{CO}_{2}$, mantendo o balanço de $\mathrm{CO}_{2}$ ou A praticamente estável.

\section{Conclusões}

1. Em condições ambientais naturais ou controladas, as cultivares de amendoim rasteiro IAC-Caiapó e Runner IAC-886 apresentam capacidades fotossintéticas iguais entre si, não sendo possível diferenciá-las por esta variável.

2. A resposta da fotossíntese em função da luz é um pouco melhor em condições ambientais ótimas (controladas).

3. As cultivares demonstram boa adaptação às variações diárias das condições ambientais que ocorrem durante o verão, apresentando fotoinibição dinâmica da fotossíntese no horário de 13 às $14 \mathrm{~h}$, quando o excesso de energia luminosa é persistente e as temperaturas foliares e as diferenças de pressão de vapor entre a folha e o ar são elevadas. 
4. O excesso de energia, nos horários de maior densidade de fluxo de fótons fotossinteticamente ativos, também é dissipado por meio do consumo de elétrons em processos alternativos.

\section{Referências}

BAKER, N.R.; ROSENQVIST, E. Applications of chlorophyll fluorescence can improve crop production strategies: an examination of future possibilities. Journal of Experimental Botany, v.55, p.1607-1621, 2004.

BAGNALL, D.J.; KING, R.W.; FARQUHAR, G.D. Temperaturedependent feedback inhibition of photosynthesis in peanut. Planta, v.175, p.348-354, 1988.

CORNIC, G.; FRESNEAU, C. Photosynthetic carbon reduction and carbon oxidation cycles are the main electron sinks for photosystem II activity during a mild drought. Annals of Botany, v.89, p.887-894, 2002. Special issue.

EDWARDS, G.E.; BAKER, N.R. Can $\mathrm{CO}_{2}$ assimilation in maize leaves be predicted accurately from chlorophyll fluorescence analysis? Photosynthesis Research, v.37, p.89-102, 1993.

GODOY, I.J. de; MORAES, S.A.; KASAI, F.S.; MARTINS, A.L.M.; PEREIRA, J.C.V.N.A.; MORAES, A.R.A.; TEIXEIRA, J.P.F. Cultivares de amendoim IAC: novas opções para o mercado de confeitaria. Campinas: Instituto Agronômico, 2003. 10p. Folder.

GODOY, I.J. de; MORAES, S.A.; SIQUEIRA, W.J.; PEREIRA, J.C.V.N.A.; MARTINS, A.L.M.; PAULO, E.M. Produtividade, estabilidade e adaptabilidade de cultivares de amendoim em três níveis de controle de doenças foliares. Pesquisa Agropecuária Brasileira, v.34, p.1183-1191, 1999.

KETRING, D.L.; BROWN, R.H.; SULLIVAN, G.A.; JOHNSON, B.B. Growth physiology. In: PATTEE, H.E.; YOUNG, C.T. (Ed.). Peanut science and technology. Yoakum: American Peanut Research and Education Society, 1982. p.411-457.

LONG, S.P.; BERNACCHI, C.J. Gas exchange measurements, what can they tell us about the underlying limitations of photosynthesis? Procedures and sources of error. Journal of Experimental Botany, v.54, p.2393-2401, 2003.

LONG, S.P.; HUMPHRIES, S.; FALKOWSKI, P.G. Photoinhibition of photosynthesis in nature. Annual Review of Plant Physiology and Plant Molecular Biology, v.45, p.633-662, 1994.

MAXWELL, K.; JOHNSON, G.N. Chlorophyll fluorescence a practical guide. Journal of Experimental Botany, v.51, p.659668, 2000.

ÖQUIST, G.; CHOW, W.S.; ANDERSON, J.M. Photoinhibition of photosynthesis represents a mechanism for the long-term regulation of photosystem II. Planta, v.186, p.450-460, 1992.

PRADO, C.H.B.A.; MORAES, J.A.P.V. Photosynthetic capacity and specific leaf mass in twenty woody species of Cerrado vegetation under field conditions. Photosynthetica, v.33, p.103-112, 1997.

RIBEIRO, R.V.; SANTOS, M.G. dos; SOUZA, G.M.; MACHADO, E.C.; OLIVEIRA, R.F.; ANGELOCCI, L.R.; PIMENTEL, C. Environmental effect on the photosynthetic capacity of bean genotypes. Pesquisa Agropecuária Brasileira, v.39, p.615-623, 2004.

VU, J.C.V. Acclimation of peanut (Arachis hypogaea L.) leaf photosynthesis to elevated growth $\mathrm{CO}_{2}$ and temperature. Environmental and Experimental Botany, v.53, p.85-95, 2005.

$\overline{\text { Recebido em } 29 \text { de março de } 2005 \text { e aprovado em } 29 \text { de março de } 2006}$ 